\title{
EVALUATION AND APPLICATION OF THE BALDWIN-LOMAX TURBULENCE MODEL IN TWO-DIMENSIONAL, UNSTEADY, COMPRESSIBLE BOUNDARY LAYERS WITH AND WITHOUT SEPARATION IN ENGINE INLETS
}

\author{
Barbara Sakowski. Douglas Darling \\ NASA - Lewis Research Center, Cleveland. OH \\ and \\ Robert L. Roach \\ Georgia Institute of Technology. Atlanta. GA \\ and \\ Allan van de Wall \\ Case Western Reserve University. Cleveland. OH
}

\begin{tabular}{|c|c|}
\hline \multicolumn{2}{|c|}{ NOMENCLATURE } \\
\hline $\mathrm{C}_{\mathrm{r}}$ & Baidwin-Lomax turbulent model parameter \\
\hline$C_{\text {ktek }}$ & Baldwin-Lomax turbulent model parameter \\
\hline $\mathrm{C}_{\mathrm{r}}$ & Skin friction coefficient \\
\hline $\mathbf{F}$ & Baldwin-Lomax outer function \\
\hline $\mathbf{M}$ & Mach number \\
\hline $\mathbf{u}$ & Velocity component in direction of core flow \\
\hline$u_{\mathrm{r}}$ & Friction velocity \\
\hline$x$ & Location in the direction of the core flow \\
\hline $\mathrm{y}$ & $\begin{array}{l}\text { Location in the direction perpendicular to the } \\
\text { core flow }\end{array}$ \\
\hline$y_{\max }$ & value of $y$ where $F$ is a maximum \\
\hline$\varepsilon$ & eddy viscosity \\
\hline$\mu$ & absolute viscosity \\
\hline$\rho$ & density \\
\hline$\tau$ & shear stress \\
\hline$\omega$ & vorticity \\
\hline SUB & PTS \\
\hline e & $\begin{array}{l}\text { core flow value, at the edge of the boundary } \\
\text { layer }\end{array}$ \\
\hline $\mathbf{w}$ & values at the wall \\
\hline
\end{tabular}

\section{INTRODUCTION}

There is a practical need to model high speed flows that exist in jet engine inlets. The boundary layers that form in these inlets may be turbulent or laminar and either separated or attached. Also. unsteady supersonic inlets may be subject to frequent changes in operating conditions. Some changes in the operating conditions of the inlets may include varying the inlet geometry, bleeds and bypasses. and rotating or translating the centerbody. In addition. the inlet may be either started or unstarted. Therefore. a CFD code, used to model these inlets, may have to be run for several different cases. Also. since the flow conditions through an unsteady inlet may be continually fluctuating, the CFD code which models these flows may have to be run over many time steps. Therefore, it would be beneficial that the code run quickly. Many turbulence models. however. are cumbersome to implement and require a lot of computer time to run. since they add to the number of differential equations to be solved to model a flow.

The Baldwin-Lomax turbulence model is a popular model. It is an algebraic. eddy viscosity model. The Baldwin-Lomax model is used in many CFD codes because it is quick and easy to implement. In this paper. we will discuss implementing the Baldwin-Lomax turbulence model for both steady and unsteady compressible flows. In addition, these flows may be either separated or attached. In order to apply this turbulence model to flows which may be subjected to these conditions, certain modifications should be made to the original Baldwin-Lomax model. We will discuss these modifications and determine whether the Baldwin-Lomax model is a viable turbulence model that produces reasonably accurate results for high speed flows that can be found in engine inlets.

\section{MODIFIED BALDWIN-LOMAX TURBULENCE MODEL}

Vishal and knight [I] suggest several moxifications to the Baldwin-Lomax turbulence model. Of these. four modifications are most significant. These four modifications were applied to the "original" Balkwin-I (nmar turbulence model for two dimensional compressible steady and unsteady flows that may or may not experience separation. These modifications are discussecl in the following sections. This paper will then evaluale these modifications as applied to two cases oullined in a later section. 


\section{Modiffcation 1: $\mathrm{C}_{\mathrm{cp}}$ and $\mathrm{C}_{\mathrm{klet}}$}

The first modification made to the Baldwin-Lomax turbulence model was to the parameters $C_{r p}$ and $C_{k \text { kiter }}$. The original model proposed by Baldwin and Lomax [2] treated these parameters as constants; a value of 1.6 was used for $C_{\mathrm{Tp}}$ and .3 was used for $C_{\text {kleb. }}$. However, Visbal and Knight [1] suggested these parameters are not constants but are. in fact. dependent on the core flow Mach number and may vary by a factor of two over the Mach number range $0<\mathrm{M}_{e}<3$. In addition, Granville [3] suggested. if a pressure gradient is present in the flow. these parameters should also be modified to include the effect of the pressure gradient. He proposed the following relationships for $\mathrm{C}_{\mathrm{p} p}$ and $\mathrm{C}_{\mathrm{kleb}}$ :

$$
\begin{gathered}
C_{k 1 \odot b}=\frac{2}{3}-\frac{0.01312}{(0.1724+\beta)} \\
C_{C p}=\frac{\left(2-4 C_{k 1 a b}\right)}{\left(2 C_{k 1 e b}-\left(2-3 C_{k 1 a b}+C_{k 1 a b}{ }^{3}\right)\right)}
\end{gathered}
$$

where $\beta$ is the modified Clauser pressure gradient parameter defined as:

$$
\beta=\frac{y_{\max }}{u_{\tau}} \frac{d u_{e}}{d x}
$$

The parameter $y_{\max }$ is used as a length scale, it is the location where the Baldwin-Lomax outer function, $F$, has the largest value. This outer function will be described in Modification 3.

\section{Modification 2: Incorporate Upstream Turbulence History Effects}

A second modification made to the Baldwin-Lomax model used by Visbal and Knight [1] was to include upstream turbulence history effects, according to a method proposed by Shang and Hankey. The eddy viscosity was modified in regions with an adverse pressure gradient. The Baldwin-Lomax eddy viscosity, $\varepsilon$, was relaxed at a streamwise location, $x$, according to the following relation:

$$
e^{\prime}=e+\left(e_{a}-e\right) e^{-\left(x-x_{e}\right) / \lambda}
$$

where $\varepsilon^{\prime}$ is the modified eddy viscosity. $x_{c}$ is the streamwise location where the pressure gradient becomes adverse. $\varepsilon_{\mathrm{n}}$ is the Baldwin-Lomax eddy viscosity at location $x_{a}$. and $\lambda$ is the relaxation length equal to the boundary layer thickness at $x_{1}$. This relation is valid for both the inner and outer regions of the flow. Equation 4 is used to modify the eddy viscosity when the flow experiences an adverse pressure gradient. An additional modification was be made to the relaxation length. The relaxation length was taken to be a factor times the incoming boundary layer thickness at the streamwise location just before the pressure gradient becomes adverse. This multiplying factor is discussed later in this paper.

\section{Modification 3: The Outer Function F}

A third modification that should be made to the Baldwin-Lomax model was also suggested by Visbal and Knight. They found that the outer function. F. used in the Baldwin-Lomax model, was not a suitable length scale in regions where the flow was separated or nearly separated. This is due to the presence of multiple maxima. or peaks. in the $F$ function. The outer function. $F$. is defined as follows:

$$
F=y|\omega| D
$$

where $D$ is the Van Driest damping factor defined as:

$$
D=1-e^{\left(\frac{-y \sqrt{P_{V} \mid \tau_{W}} \mu_{W}}{26}\right)}
$$

This function. F. will typically have two significant maxima in the $y$-direction. It is necessan to choose the correct maximum value of $F$. $F_{\text {inax }}$. for each $y$ location. Typically. the correct peak is the one furthest awas from the wall. The value of $y_{\text {ma: }}$ chosen. should he at this outer peak. When the boundary laser is not separated or nearly separated. the outer peak will have the 
larger value of F. However, when the boundary layer is nearly separated, the inner peak may have the larger value of F. However, the outer peak should still be chosen for $F_{\max }$ and $y_{\max }$. The peak closer to the wall will have smaller normal locations, $y$. Choosing a peak that is not the furthest from the wall may yield an abrupt and unrealistic reduction in the calculated outer eddy viscosity, due to the reduction in the predicted $y_{\max }$

There is another problem in determining the maximum value of the $F$ function in regions of strong interaction such as separation. In regions of separation and downstream of separation. the outermost peak of the $F$ function tends to disappear and the innermost peak tends to move further away from the wall. This may cause large streamwise variations in the normal location of $\mathrm{F}_{\max }$, which would in turn yield unrealistic variations in the calculated outer eddy viscosity. A way to overcome this problem is to hold the value of the normal location. $y_{\max }$, constant for $x$ locations at separation and at all streamwise locations downstream of separation. The value of $y_{\max }$ used should be that of a streaniwise location several $x$ stations upstrean of separation. $F_{\max }$ is still calculated for each station downstream of separation, however it is calculated at the constant $y_{\max }$ (from upstream of separation) rather than normal location. $y$, where $F$ is a maximum.

\section{Modification 4: The Van Driest Damping Factor}

The final modification made to the Baldwin-Lomax model was to the Van Driest damping factor, $D$ (equation 6$)$. In separated regions, the wall shear stress, $\tau_{w}$, approaches zero. As Visbal and Knight pointed out, this causes an unrealistic reduction in magnitude of the Van Driest damping factor, which in turn causes an unrealistic reduction in the calculated inner eddy viscosity. A way to avoid this problem is to use the total shear stress at the given normal location, $y$. to calculate $D$. This is used only for regions where there is separation. The total shear stress is determined using the sum of the laminar viscosity and the turbulent eddy viscosity. However, at a given streamwise station and normal grid location. $D$ is itself used to determine the turbulent viscosity. Therefore. the known turbulent eddy viscosity from the previous streamwise location and same normal grid location is used to determine the total shear stress to calculate $\mathrm{D}$.

\section{RESULTS AND DISCUSSION}

The above modifications were made to the BaldwinLomax turbulence model. These modifications were themselves "modified" to better match experimental data for high speed flows. Two cases (Table 1) were examined to obtain numerical results for high speed flows.

For CASE 1, the Baldwin-Lomax model was incorporated in an unsteady boundary layer algorithn that used a given inviscid core solution. The Large Perturbation Inlet Code (LAPIN), was the code used to determine the inviscid core flow solution. The forms of the boundary layer equations used in the algorithm were the uncoupled. unsteady, compressible. parabolized Navier-Stokes (PNS) equations by Roach, et al [4]. In this case, the boundary layer algorithm and LAPIN were interactive. The turbulent boundary layer in LAPIN was subjected to both favorable and adverse pressure gradients. and on occasion experienced separation. Figure 1 shows the geometry of the NASA-LeRC $40-60$ inlet used for Case 1. For CASE 2. the modified Baldwin-Lomax model was incorporated in an uncoupled. steady, compressible. boundary layer algorithm utilizing the PNS equations by Roach. et al. In this case the boundary layer was not interactive with the core flow. The inviscid core flow was prescribed based on the experimental results of Lewis. et al [5]. The turbulent boundary layer in this second case experienced no separation but was subjected to both adverse and favorable pressure gradients. Figure 2 shows the core flow Mach number distribution as a function of $x$ used for Case 2. In this case, calculations were started using a zero pressure gradient profile with a Reynolds number, based on momentum thickness. of about 4800 at $x=11.5$ inches downstream of the leading edge.

\section{Table 1 Summary of Cases}

\section{CASE 1}

Unsteady

Compressible

Interactive Core Flow

Core Flow from LAPIN

Bleeds and Bypasses

\section{CASE 2}

Steady

Compressible

Non-interactive Core Flow

Core Flow Prescribed

No Bleeds. No Bypasses 


\section{Effects of Modifying $C_{c p}$ and $C_{k l e t}$}

In determining the effects of varying $\mathrm{C}_{\mathrm{ap}}$ and $\mathrm{C}_{\mathrm{kteb}}$ (modification 1) on the Baldwin-Lomax turbulence into the model. Although modifications 3 and 4 will be discussed in greater detail in a later section, it was necessary to incorporate them at this point so the empirical relations for the parameters $\mathrm{C}_{\mathrm{r}}$ and $\mathrm{C}_{\mathrm{kscb}}$ would be compatible with them.

The original Baldwin-Lomax values of $C_{\mathrm{ct}}=1.6$ and $\mathrm{C}_{\mathrm{k} k \mathrm{teb}}=.3$ predicted $\mathrm{C}_{\mathrm{f}}$ values that were significantly smaller than the experimental $C_{f}$ values for the flow described in Figure 2. (CASE 2). Unfortunately, using equations $1-3$ in the Baldwin-Lomax turbulence model cantly smaller than the experimental $C_{f}$ values. Therefore, another equation needed to be determined to relate the parameters $C_{\mathrm{cT}}$ and $\mathrm{C}_{\mathrm{kjeb}}$ to the core flow Mach number and pressure gradient. An equation was obtained based upon the experimental $C_{r}$ values from CASE 2. It was found that the following empirical relation:

$$
C_{C P}=1.6(1+M)
$$
model. modifications 3 and 4 were already incorporated for CASE 2 also predicted $C_{r}$ values that were signifi-

The above relation for $\mathrm{C}_{\mathrm{cp}}$ was incorporated into the turbulence model for CASE 1, an unsteady, compressible flow in a NASA-LeRC 40-60 inlet. Figure 4 shows a plot of the ratio of the local static pressure to the free stream static pressure versus position in the inlet. Using Equation 7 to determine $C_{c r}$ as a function of Mach number in the favorable pressure gradient region of the flow, pushes the shock wave upstream of the shock wave position determined from experimental data. Thus a further modification to the relationship between $C_{\text {ip }}$ and Mach number was necessary. This is because Equation 7 was empirically developed for a Mach number range from 2.5 to 4 . The core flow from CASE I goes from subsonic speeds to about a Mach number of about 2.5. Equation 5 must be modified to incorporate a $C_{\mathrm{tp}}$ distribution with Mach number that is compatible for a Mach number range from 0 to 2.5. It was determined that for lower Mach numbers. below about 2. $C_{\mathrm{cp}}$ needed to be significantly smaller than the value it had at Mach numbers greater than 2.5. The following relationship for $C_{p p}$ as a function of Mach number was developed to satisfy all these conditions:

$$
C_{C P}=4.6+\frac{6.8}{\pi} \arctan (2.0(M-2.5))
$$

worked well in flow regions with no pressure gradients and flow regions with favorable pressure gradients. However. in regions of adverse pressure gradients, using the original value of $\mathrm{C}_{\mathrm{rp}}=1.6$ compared better to the experimental results than using equation 7 . Varying the constant $\mathrm{C}_{\text {veh }}$ did not significantly effect a change in $\mathrm{C}_{\mathrm{r}}$, so it was left at its original value of .3 . Figure 3 illustrates $\mathrm{C}_{\mathrm{r}}$ as a function of $\mathrm{x}$ for CASE 2 for the following: (1) the experimental results from Lewis et al., (2) the turbulence model from Cebeci and Smith [6], (3) the Baldwin-Lomax turbulence model with $C_{\mathrm{cr}}=1.6$. and (4) the Baldwin-Lomax turbulence model using equation 7 to determine $C_{c p}$ in regions without an adverse pressure gradient. Figures 3 shows that by using the modified equation for $C_{c p}$ (equation 7) the model compares well to the experimental data and to the model of Cebeci and Smith. However the Baldwin-Lomax model, just like the Cebeci and Smith model. tends to underpredict the skin friction values beyond the adverse region. This is consistent with the conclusions made by Visbal and Knight. All the turbulence models they tested failed to predict the rapid recovery of boundary layers after adverse regions, such as downstream of the region of reattachment after shock waves.
Figure 5 shows a plot comparing Equations 7 and 8. Equation 8 yields the general trend of $\mathrm{C}_{\mathrm{cr}}$ as function of Mach number for a Mach number range from 0 to 4 . Equation 7 only captures the general trend for Mach numbers greater than 2.5. Figure 4 illustrates that incorporating equation 8 into the Baldwin-Lomax model. pushes the shock wave closer to the position determined from the experimental data. Equation 8 was also validated for CASE 2. Its use in the turbulence model as a substitute for equation 7 produced negligible changes in the skin friction coefficient.

It should be noted that if CASE 1 is run for a constant value of $C_{c p}=1.6$, the pressure distribution obtained is practically identical to the pressure distribution obtained when Equation 8 is used to determine $\mathrm{C}_{\mathrm{c} r}$. This is because the core flow Mach number range is from 4 to 2 . In this range. $C_{\text {ti }}$ on the most part averages a value of about 1.6. thereby giving practically the same pressure distribution that would be obtained it $C_{c}$ was held constant at the value of 1.6. Equation 8 inly satisfies the need of transitioning $C_{C}$ to significantls higher values. i.e. 4 to 8 , for $2.5<M_{\text {. }}<4$. 


\section{Effect of Adding Upstream Turbulence History}

Equation 4 was incorporated into the Baldwin-Lomax turbulence model for CASE 2. As stated previously (modification 2 ), the relaxation length, $\lambda$, (of equation 4) was multiplied by factor. This factor was varied to determine its effect on the skin friction coefficient. The factors used were 1, 10. and 25. Figure 6 shows a plot of $C_{1}$ versus $x$ for the relaxation length multiplied by the factors 1,10 , and 25 . These results are based upon the modified relation for $C_{r r}$ (equation 8 ). Also included on figure 6 are the experimental results of Lewis et al.. and the results from the numerical scheme of Cebeci and Smith. These results indicate that multiplying the relaxation length by 1 yields a skin friction distribution that compares very well to the experimental data. However using a multiplication factor of 1 yields the same results as not incorporating equation 4 in the Baldwin-Lomax turbulence model to modify the eddy viscosity in the adverse region of the flow. This eddy viscosity modification was also incorporated in the region downstream the adverse flow region where the pressure gradient becomes favorable again for the same cases illustrated in figure 6 . The turbulence model respectively yielded skin friction results that were no different from the results illustrated in figure 6 .

The same qualitative results were obtained for CASE I when the relaxation multiplication factors were varied Using multiplication factors of 10 and 25 caused the core flow pressure to drop below the experimental values in the throat area of the inlet, see figure 7 . This region is just beyond the adverse flow region which modifies the turbulent eddy viscosity according to equation 4. Using a multiplication factor of 1 yields a core flow pressure distribution in the throat that matches the experimental results. This pressure distribution for a multiplication factor of 1 is also the same as the pressure distribution obtained when no modification to the eddy viscosity is made (equation 4 is not used). The results of CASE 1 and CASE 2 indicate that the use of equation 4 is not necessary to obtain results that closely correspond to experimental and numerical results. In fact, use of equation 4 with the relaxation length multiplied by a factor greater than 1 produce results which deviate from experimental data.

\section{Effect of Modifying the Outer Function F}

It was stated in a previous section that the outer function. F, had already been modified so as to determine a compatible equation for $\mathrm{C}_{\mathrm{c} p}$ as a function of $\mathrm{Mach}$ number. This modification insured that the conect maxi- mum was chosen at each $x$-location. In flow regions near separation. the outer function. F, may have multiple maxima. The correct maximum to choose is furthest from the wall. However, in flow regions that are separated and the regions where reattachment occurs, these maxima become ambiguous. Calculating $F_{\max }$ and its respective $y_{\max }$ for $x$ locations at separation and downstream of separation becomes inappropriate. This is because $F_{\max }$ is no longer a valid velocity scale. and $y_{\max }$ is no longer a valid length scale. Visbal and knight determined that downstream of separation the outer maxima of $F$ tend to disappear while the inner maxima of $F$. or the maxima of F closer to the wall. move away from the surface to a new equilibrium position. Visbal and Knight recommend holding $\mathrm{y}_{\max }$ constant at $\mathrm{x}$ locations through separation and downstream of separation. For CASE 1. this constant value of $y_{\max }$ was taken to be the value of $y_{\max }$ three grid locations, in the streamwise direction, upstream of the separation point. Although $y_{\max }$ was no longer calculated but held at the same value for each $x$ station at separation and downstream of separation, the outer function $F$ was still calculated. In this case. the value of the outer function at each $x$ location at separation and downstream of separation was no longer the maximum of the outer function. It was simply the calculated value of the outer function for the given $\mathrm{y}_{\max }$ and $\mathrm{x}$ location.

It was interesting to see what would happen if for CASE 1 . both $F_{\max }$ and $y_{\max }$ were held constant at separation and downstream of separation. The values of $F_{\max }$ and $y_{\max }$ would be respectively the values of $F_{\max }$ and $y_{\max }$ three grid locations upstream the separation point. Furthermore, it was also interesting to see what would happen if neither $F_{\max }$ nor $y_{\max }$ were held constant at separation and downstream of separation, but calculated respectively. The results are shown in Figure 8 . These results indicate that continuing to calculate new values for $F_{\max }$ and $y_{\max }$ at points of separation and downstream of separation, positions the shock wave where the experimental position of the shock wave is. These resulis also correspond very closely to the results of the case when only $y_{\max }$ is held constant through separation and downstream of separation. while continuing to calculate the value of the outer function at this value of $y_{\text {nm }}$ (as described above). This case is also illustrated in Figure 8. Finally. holding $F_{\text {ma: }}$ as well as ? mat at their respective values three grid locations in the stleanmise direction. upstream separation. pushes the shock wave eten further downstream of the experimental observation. Thus the results from CASE 1 indicate that thete is not a significant difference in holding $y_{\text {ina.. }}$ constant though separation while calculating its respective outer function 
value. $F$, and calculating both $y_{\max }$ and $F_{\max }$ through separation.

\section{Effect of modifying the Van Driest Damping Factor}

In regions in and near separation the wall shear stress is close to zero. which in turn causes the $V$ an Driest damping factor to become sniall. Consequently there is a reduction in the computed eddy viscosity. Visbal and Knight recommend using the local shear stress instead of the wall shear stress in equation 6 near regions of separation and reattachment.

Figure 9 shows the static pressure distribution versus $x$ for CASE 1 . Only in the region of flow reattaclment is there a slight difference in using the local shear stress as opposed to the wall shear stress in equation 6 . For the most part the global effects (e.g. core flow pressure. shock position) of using the local shear stress instead of the wall shear stress was not very significant. Using the local shear stress in equation 6 was more significant in determining skin friction coefficients in region of reattachment.

This effect is illustrated in figure 10. Figure 10 is a plot of the skin friction coefficient versus $x$ location for CASE 1. Note that in the region where there is flow reattachment, $C_{r}$ is slightly reduced when the wall shear stress is used in equation 6.

\section{CONCLUSIONS}

The Baldwin Lomax turbulence model was modified to model unsteady. compressible flows with and without separation. Modifications were made to the parameters $\mathrm{C}_{\mathrm{cp}}$ and $\mathrm{C}_{\mathrm{k} \cdot \mathrm{b}}$. as well as the calculations of the eddy viscosity, the outer function $F$ and the van Driest damping factor. The most significant modification made to the Baldwin-Lomax model was in making $C_{c}$ a function of both Mach number and pressure gradient. This modification was extremely important in matching numerical results to experimental data. The other modifications did not significantly improve the numerical results as did the modification to $\mathrm{C}_{\mathrm{t} T}$.

The value of $C_{\mathrm{cp}}$ used should be different in regions of adverse pressure gradients than regions of favorable pressure gradients. In addition. there were two noticeable regions of Mach number for using $\mathrm{C}_{\mathrm{tr}}$. When the edge Mach number is below about 2.5. the original value of $C_{41}=1.6$ works fairly well. For edge Mach numbers greater than about $2.5 . \mathrm{C}_{\mathrm{t} T}$ should be significantly larger. Equation 8 seemed to work well for both ranges when the pressure gradient was not adverse Altering $C_{k k+1}$ did not significantly change the results of the model. so the original value of $\mathrm{C}_{\mathrm{kk} \mathrm{t}}=0.3$ was used.

Modifying the eddy viscosity to account for upstream turbulence effects in the regions with adverse pressure gradients did not affect the results of the model. However. if the relaxation length scale was increased. the predicted results deviated from the experimental results.

Other modifications to the Baldwin-Lomax turbulence model included. holding $y_{\text {max }}$ constant in regions of separation for calculating $F_{m \times r}$. and using the local total shear stress, instead of the wall shear stress. to calculate the van Driest damping factor in regions of separation. Both of this modifications improved the accuracy of the model.

\section{REFERENCES}

1. M. Visbal. D. Knight. "The Baldwin-Lomax Turbulence Model for Two-Dimensional Shock-Wave/Boundary-Layer Interactions." AlAA Joumal Vol. 22. NO.7. July 1984. pp $921-928$.

2. B.S. Baldwin. H. Lomax. "Thin Layer Approximation and Algebraic Model for Separated Turbulent Flows." AIAA 78-2.57. 1978.

3. P.S. Granville. "Baldwin-Lomax Factors for Turbulent Boundary Layers in Pressure Giradients." AIAA Journal Vol. 25. NO. 12. December 1987, pp 1624-1627.

4. R.L. Roach. C. Nelson. B.A. Sakowski. D.D. Darling. A.G. van de Wall. "A Fast. Uncoupled. Compressible. Two-Dimensional. Unsteady Boundary Layer Algorillum with Separation for Engine Inlets." AIAA 92-30182. 1992.

5. J.E. Lewis. R.L. Gran. T. Kubola. "An Experiment on the Adiabatic Compressible Turbulent Boundary Layer in Adverse and Farorable Pressure Gradients," I. Finicl Mle. chanics 1072. Vol. 51. Tar1 4. pe 0.51072.

T. Cebeci. A.M. S Smill. "Analysis "If Tubulent Boundary Layers." Academic Press. New York 1974. 
$7 . \quad$ R.W. Cubbison, E.T. Meleason, D.F. Johnson. "Performance Characteristics from Mach 2.58 to 1.98 of an Axisymmetric MixedCompression Inlet System with 60-Percent Internal Contraction," NASA TM X-1739. 1969.

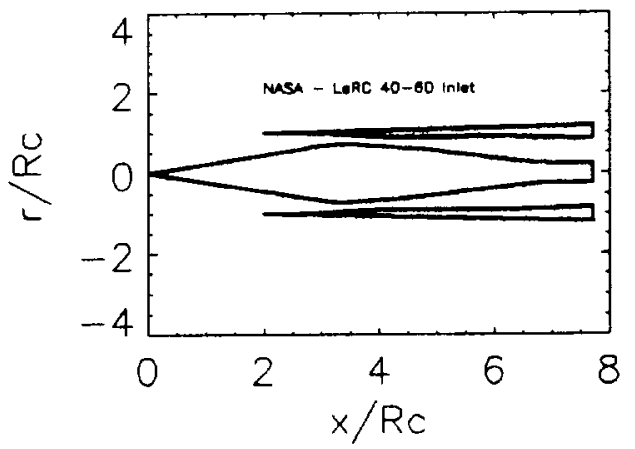

Figure 1 Geometry of the NASA-LeRC 4060 supersonic inlet, used for CASE 1.

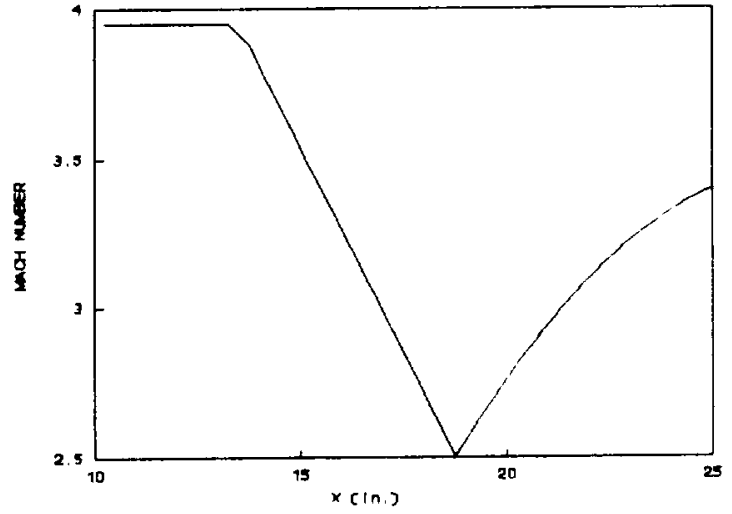

Figure 2 Mach number versus Position downstream, from experiment by Lewls, et al. [5], used for CASE 2.

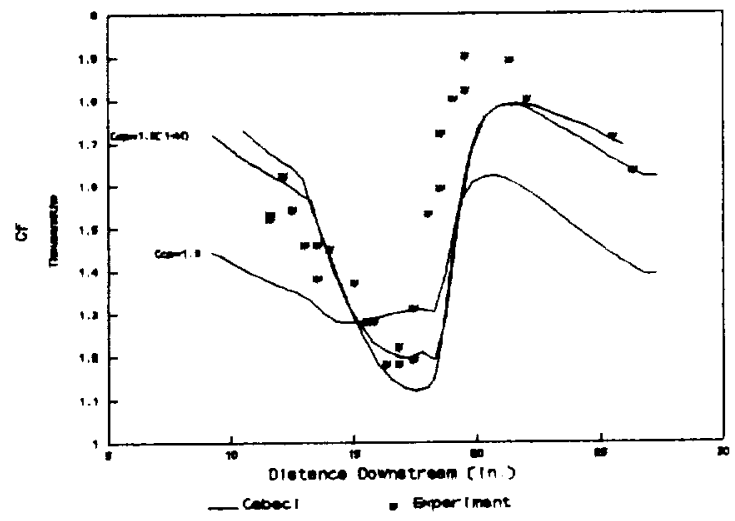

Figure 3 CASE 2: $\mathrm{C}_{\mathrm{f}}$ versus position for $C_{c p}=1.6, C_{c p}=f\left(M_{e}\right.$. pressure gradient) conpared with a model of Cebeci [6] and experiments of Lewis, et al. [5]. 


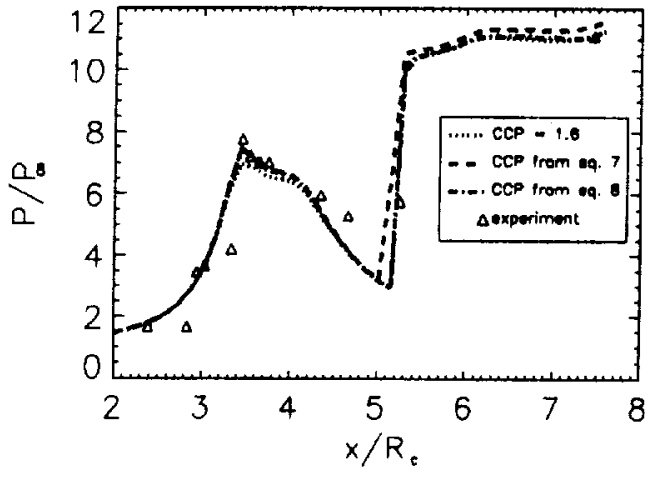

Figure 4

CASE 1: The ratio of static pressure to upstream pressure versus position for $C_{r p}=1.6$ and $C_{c p}$ calculated from equation 7 and from equation 8. Experimental results from Cubbison, et al. [7].

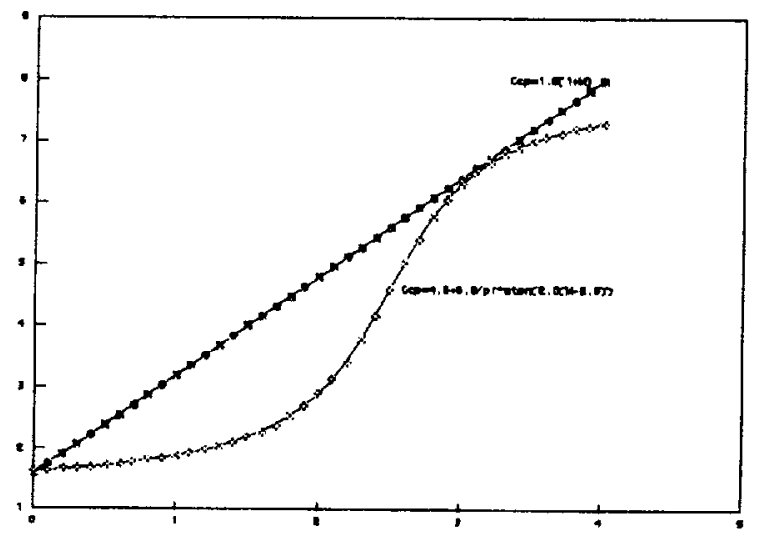

Figure $5 \quad \mathrm{C}_{\mathrm{p}}$ (vertical axis) as a function of Mach number (horizontal axis) following equation 7 and equation 8.



Figure 6

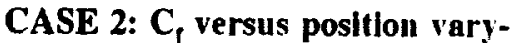
ing the relaxation length for upstream turbulence effects. Relaxation length, $\lambda$, is multiplled by 1 , 10 , and 25.

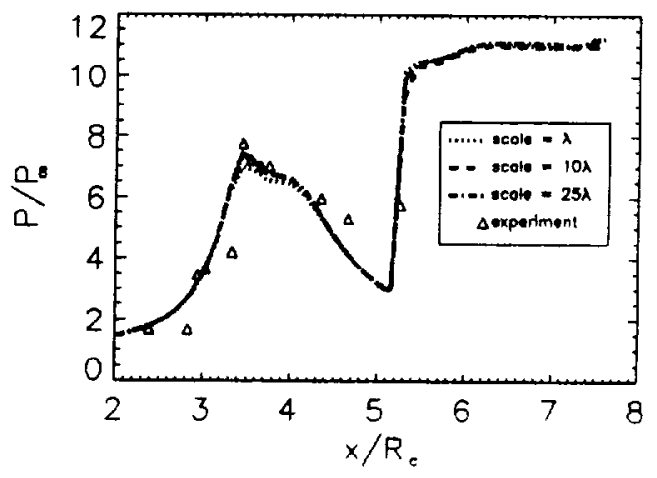

Figure 7

CASE 1: Predicted ratio of local static pressure to upstream static pressure versus position, varying the relaxation length. The relaxatlon length is multiplied by 1.10. and 25. Experimental obsernntions from Cubbison et al. [7]. 


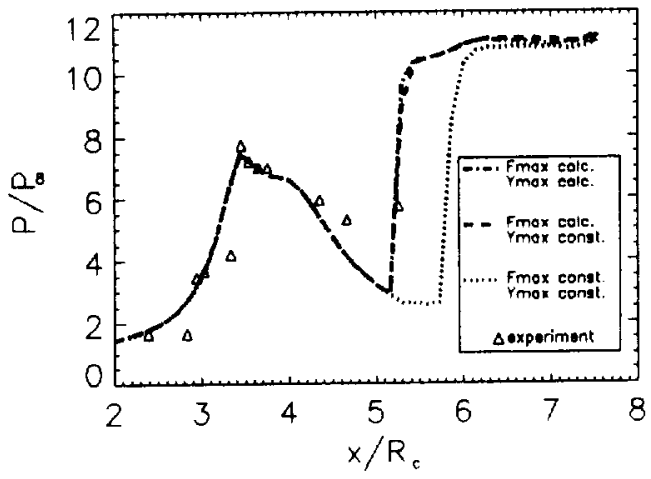

Figure 8 CASE 1: Predicted ratio of local static pressure to upstream static pressure versus position, varying the logic of $y_{\max }$ and $F_{\max }$ calculations. Experimental results are taken from Cubbison, et al. [7].

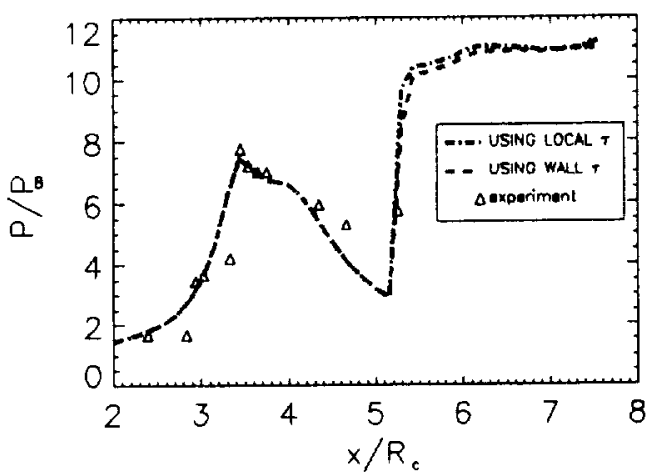

Figure 9 CASE 1: Predicted ratio of local static pressure to upstream static pressure versus position, using local and wall shear stress to calculate van Driest damping factor (equation 6). Experimental results from Cubbison et al. [7].

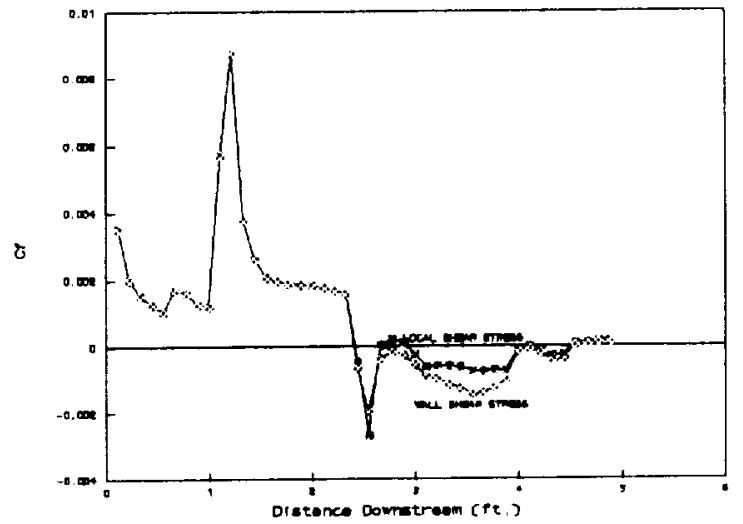

Figure 10 CASE 1: $\mathbf{C}_{\mathrm{r}}$ versus position, using local and wall shear stress to calculate van Driest damping factor (equation 6). 


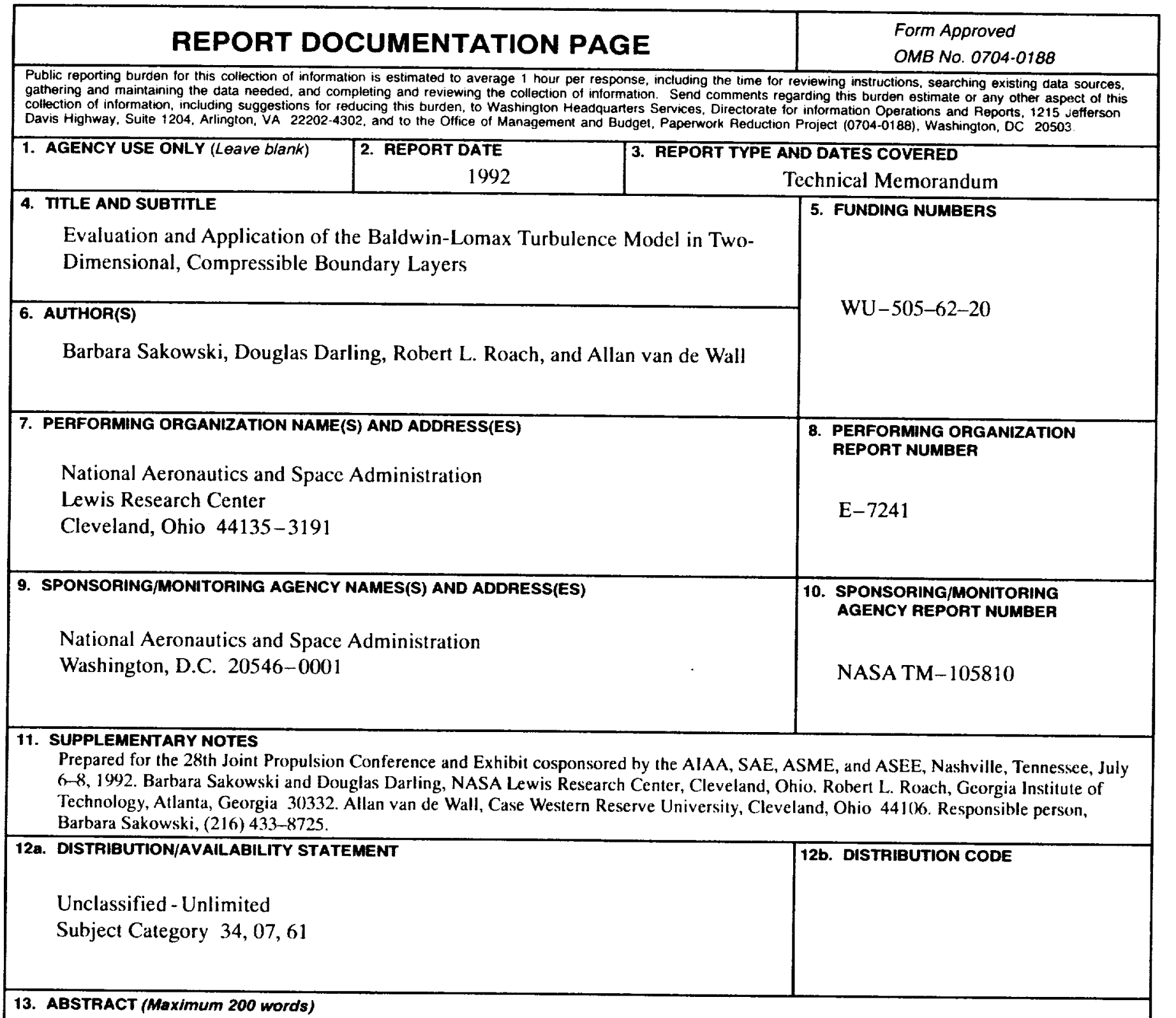

There is a practical need to model high speed flows that exist in jet engine inlets. The boundary layers that form in these inlets may be turbulent or laminar and either separated or attached. Also, unsteady supersonic inlets may be subject to frequent changes in operating conditions. Some changes in the operating conditions of the inlets may include varying the inlet geometry, bleeds and bypasses, and rotating or translating the centerbody. In addition, the inlet may be either started or unstarted. Therefore, a CFD code, used to model these inlets, may have to run for several different cases. Also, since the flow conditions through an unsteady inlet may be continually fluctuating, the CFD code which models these flows may have to be run over many time steps. Therefore, it would be beneficial that the code run quickly. Many turbulence models, however, are cumbersome to implement and require a lot of computer time to run, since they add to the number of differential equations to be solved to model a flow. The Baldwin-Lomax turbulence model is a popular model. It is an algebraic, eddy viscosity model. The Baldwin-Lomax model is used in many CFD codes because it is quick and easy to implement. In this paper, we will discuss implementing the Baldwin-Lomax turbulence model for both steady and unsteady compressible flows. In addition, these flows may be either separated or attached. In order to apply this turbulence model to flows which may be subjected to these conditions, certain modifications should be made to the original Baldwin-Lomax model. We will discuss these modifications and determine whether the Baldwin-Lomax model is a viable turbulence model that produces reasonably accurate results for high speed flows that can be found in engine inlets.

14. SUBJECT TERMS

Turbulence model; Baldwin-Lomax; Compressible flow; Separated flow; Engine inlet

\begin{tabular}{|c|c|}
\hline $\begin{array}{c}\text { 17. SECURITY CLASSIFICATION } \\
\text { OF REPORT } \\
\text { Unclassified }\end{array}$ & $\begin{array}{c}\text { 18. SECURITY CLASSIFICATION } \\
\text { OF THIS PAGE } \\
\text { Unclassified }\end{array}$ \\
\hline
\end{tabular}

19. SECUATY CLASSIFICATION OF ABSTAACT Unclassified
15. NUMBER OF PAGES 10 16. PRICE CODE $\mathrm{A} 02$

\section{LIMITATION OF ABSTRACT}

